\title{
Real-world effectiveness of post- trastuzumab emtansine treatment in patients with HER2-positive, unresectable and/or metastatic breast cancer: a retrospective observational study (KBCSG- TR 1917)
}

Takahiro Nakayama ${ }^{1 *}$ D, Tetsuhiro Yoshinami ${ }^{2}$, Hiroyuki Yasojima ${ }^{3}$, Nobuyoshi Kittaka ${ }^{1}$, Masato Takahashi ${ }^{4}$, Shoichiro Ohtani ${ }^{5,6}$, Seung Jin Kim² ${ }^{2}$, Hiroyuki Kurakami ${ }^{7}$, Naoko Yamamoto ${ }^{7}$, Tomomi Yamada',

Takehiko Takata ${ }^{8}$ and Norikazu Masuda ${ }^{3}$ (D)

\begin{abstract}
Background: Trastuzumab emtansine (T-DM1) is a second-line standard therapy for patients with human epidermal growth factor receptor 2 (HER2)-positive metastatic breast cancer. Evidence regarding post-T-DM1 treatments is currently lacking. We evaluated the effectiveness of post-T-DM1 drug therapy in patients with HER2-positive, unresectable and/or metastatic breast cancer.

Methods: In this multicenter, retrospective, observational study, real-world clinical data of female patients with HER2-positive breast cancer who had a history of T-DM1 treatment were consecutively collected from five sites in Japan. We investigated the effectiveness of post-T-DM1 therapy by evaluating the real-world progression-free survival (rwPFS), time to treatment failure (TTF), overall survival (OS), objective response rate (ORR), and clinical benefit rate (CBR). Tumor response was assessed by investigators according to Response Evaluation Criteria in Solid Tumors (RECIST version 1.1) guidelines. Subgroup and exploratory analyses according to background factors were also undertaken.
\end{abstract}

\footnotetext{
* Correspondence: taqnakayama@gmail.com

${ }^{1}$ Department of Breast and Endocrine Surgery, Osaka International Cancer Institute, 3-1-69, Otemae, Chuo-ku, Osaka 541-8567, Japan

Full list of author information is available at the end of the article
}

(c) The Author(s). 2021 Open Access This article is licensed under a Creative Commons Attribution 4.0 International License, which permits use, sharing, adaptation, distribution and reproduction in any medium or format, as long as you give appropriate credit to the original author(s) and the source, provide a link to the Creative Commons licence, and indicate if changes were made. The images or other third party material in this article are included in the article's Creative Commons licence, unless indicated otherwise in a credit line to the material. If material is not included in the article's Creative Commons licence and your intended use is not permitted by statutory regulation or exceeds the permitted use, you will need to obtain permission directly from the copyright holder. To view a copy of this licence, visit http://creativecommons.org/licenses/by/4.0/. The Creative Commons Public Domain Dedication waiver (http://creativecommons.org/publicdomain/zero/1.0/) applies to the data made available in this article, unless otherwise stated in a credit line to the data. 
Results: Of the 205 patients who received T-DM1 treatment between 1 January 2014 and 31 December 2018, 128 were included in this study. Among the 128 patients analyzed, 105 (82\%) patients received anti-HER2 therapy and 23 (18\%) patients received regimens without anti-HER2 therapy. Median (95\% confidence interval [CI]) rwPFS, TTF, and OS were 5.7 (4.8-6.9) months, 5.6 (4.6-6.4) months, and 22.8 (18.2-32.4) months, respectively. CBR and ORR $(95 \% \mathrm{Cl})$ were $48 \%(38.8-56.7)$ and $23 \%(15.1-31.4)$, respectively. Cox-regression analysis showed that an ECOG PS score of 0 , a HER2 immunohistochemistry score of 3+, recurrent type, $\geq 12$ month duration of T-DM1 therapy, and anti-HER2 therapy were independent variables for rwPFS. An exploratory subgroup analysis of regimens after T-DM1 showed that those with anti-HER2 therapy had a median rwPFS of 6.3 and those without anti-HER2 therapy had a median rwPFS of 4.8 months.

Conclusions: In the real-world setting in Japan, several post-T-DM1 regimens for patients with unresectable and/or metastatic HER2-positive breast cancer, including continuation of anti-HER2 therapy, showed some effectiveness; however, this effectiveness was insufficient. Novel therapeutic options are still needed for further improvement of PFS and OS in later treatment settings.

Trial registration: UMIN000038296; registered on 15 October 2019.

Keywords: Retrospective observational study, HER2-positive, Unresectable and/or metastatic breast cancer, KBCSGTR 1917, T-DM1/trastuzumab emtansine

\section{Background}

Human epidermal growth factor receptor 2 (HER2) is a growth factor receptor gene that is amplified in approximately $15-20 \%$ of breast cancers, and HER2 protein overexpression on the plasma membrane of tumor cells reportedly correlates with a poor prognosis [1-6].

Trastuzumab, a HER2-targeting monoclonal antibody, was approved in 1998 and has improved outcomes in patients with HER2-positive breast cancer [7, 8]. Following trastuzumab approval, other HER2-targeted drugs have subsequently been approved for use in these patients, including lapatinib $[9,10]$ and pertuzumab $[11$, 12]. These therapies have been reported to prolong progression-free survival (PFS) and overall survival (OS), and to be more efficacious than conventional chemotherapies [11-16]. Based on the outcomes of the CLEOPATRA trial [12], pertuzumab + trastuzumab + taxane is currently recommended as first-line therapy in HER2positive metastatic breast cancer [17].

The EMILIA trial investigated the use of trastuzumab emtansine (T-DM1) as a second-line therapeutic to follow treatment with trastuzumab and a taxane [13]. Additionally, the TH3RESA trial demonstrated that patients who had previously been treated with two or more regimens experienced an increase in objective response rate (ORR) and a prolongation of PFS and OS with T-DM1 treatment $[14,15]$. T-DM1 is now the standard of care for patients with HER2-positive metastatic breast cancer who were previously treated with trastuzumab + taxane [18]. However, the development of T-DM1 resistance, either through reduced HER2 expression, reduced TDM1 binding, or other subversive signaling abnormalities, remains a challenge [19]. For example, we previously reported several cases where tumors became
HER2-negative after T-DM1 treatment [20]. Currently, there are no established treatment options to follow TDM1 therapy that have shown adequate evidence in real-world settings. Therefore, we planned the present study to establish real-world evidence to support clinical treatment decisions. In this multicenter, retrospective observational study conducted by the Kinki Breast Cancer Study Group-Translational Research (KBCSG-TR), we aimed to examine real-world effectiveness following T-DM1 discontinuation (post-T-DM1 treatment) in patients with HER2-positive, unresectable and/or metastatic breast cancer.

\section{Methods}

\section{Study design and patient population}

The KBCSG-TR 1917 study (UMIN000038296) was a multicenter, retrospective, observational study conducted in patients with HER2-positive, unresectable and/or metastatic breast cancer. The data cut-off date for all analyses was 31 July 2019. Electronic medical records from five sites in Japan were used to identify patients who had received T-DM1 treatment (either as a single-agent or in a combination therapy regimen) between 1 January 2014 and 31 December 2018.

The inclusion criteria were as follows: women aged $\geq 20$ years at the start of post-T-DM1 treatment; pathological diagnosis of HER2-positive, unresectable and metastatic breast cancer (immunohistochemistry [IHC] $3+$, IHC $2+$ and in situ hybridization [ISH]+, or IHC not evaluated and $\mathrm{ISH}+$ ) according to the Japanese Breast Cancer Society "General rules for clinical and pathological recording of breast cancer" [21] at the time of diagnosis; and initiation of at least one line of drug therapy (anti-HER2 targeted therapy, molecular targeted 
therapy, chemotherapy, or endocrine therapy) for HER2positive, unresectable and/or metastatic breast cancer between 1 January 2014 and 31 December 2018 immediately after T-DM1 treatment discontinuation. The exclusion criteria were as follows: patient had received an approved or new investigational drug without a breast cancer indication (as defined in Japan) in any clinical trial immediately following T-DM1 treatment discontinuation; or expression (prior to the database lock) of the intention not to participate in this study using the opt-out approach.

We considered that the median PFS in the control group (treatment of the physician's choice) in the TH3RESA trial was 3.3 months [15], and thus determined that a 4-month observation period for real-world PFS (rwPFS) assessment would be sufficient.

\section{Ethics approval}

This retrospective observational study involving human participants was conducted in accordance with the ethical principles found in the Declaration of Helsinki, the Ethical Guidelines for Medical and Health Research Involving Human Subjects, and in compliance with the study protocol and all applicable local and national ethical guidelines. This study was approved by the ethic screening committee of Osaka Prefectural Hospital Organization Osaka International Cancer Institute. As a non-interventional study with no invasive procedures or human-derived specimens, informed consent was neither required nor obtained from study participants; the optout approach was employed to ensure that patients had the opportunity to refuse the registration of their information in this study.

\section{Patient registration and data collection}

Patients with breast cancer who had a history of T-DM1 treatment were identified by study investigators using the medical record search system at each study site. Once extracted, patient records were checked and those who met all of the inclusion criteria and none of the exclusion criteria were considered as study participants. Eligible patients were then consecutively registered from 3 September 2019 to 22 November 2019. Anonymized data from the medical records of all patients registered in the study were entered into the DATATRAK ONE system (DATATRAK Int., Mayfield Heights, OH, USA).

\section{Study outcomes}

Outcome assessments included rwPFS, time-totreatment failure (TTF), OS, ORR, and clinical benefit rate (CBR). rwPFS was selected as the outcome measure due to the nature of the study design (using electronic medical records), and was in line with previous, similar, analyses [22].
Tumor responses were assessed by the study investigators in patients with measurable target lesions, ideally complying with the Response Evaluation Criteria in Solid Tumors (RECIST) guidelines (version 1.1). Cancer progression was diagnosed by the attending physician at the time of treatment. In this study, priority was given to the attending physician over the study investigator's assessment of documented cancer progression based on RECI ST (version 1.1). rwPFS was counted from the start date of post-T-DM1 drug therapy to the date of the first documented cancer progression (after the start date of post-T-DM1 drug therapy) or the date of all-cause death, whichever occurred first. The last date of documented rwPFS was the earliest occurring date of the following: post-treatment start date, last visit date, or data cut-off date. TTF was defined as the time from the start date of post-T-DM1 drug therapy to the date of the decision on treatment discontinuation by the attending physician (including disease progression and treatment toxicity). OS was defined as the time from the start date of post-T-DM1 drug therapy to the date of death from any cause. ORR was defined as the percentage of the patient population with the best tumor response (complete response $[\mathrm{CR}]$ or partial response $[\mathrm{PR}]$ ). The CBR was defined as the percentage of the patient population whose best tumor response was CR or PR or who continued treatment for at least 6 months (from the start date of post-T-DM1 drug therapy). Additional details can be found in Additional File 1.

\section{Statistical analysis}

The sample size was set to allow each participating institution to register all eligible patients during the study period. Prior to starting the study, we conducted a survey at each study site and from this were able to estimate the number of patients considered feasible to enroll during the study period. Assuming that five facilities could enroll 20 patients per facility, we expected a total enrolment of 100 patients.

Descriptive statistics were calculated for summaries of patient characteristics. The median survival for rwPFS, TTF, and OS was calculated using the Kaplan-Meier method to estimate the survival curve and the log-rank test to compare the groups; point estimates of survival rates at $6,12,18,24$, and 36 months, as well as their 95\% confidence intervals (CI) were calculated using Greenwood's formula. For rwPFS, univariate and multivariate Cox-regression analyses were performed as exploratory analysis; for selection of variables in the multivariate analysis, "previous pertuzumab" and "regimens after TDM1" were entered using the forced entry method, whereas other variables were selected using the stepwise method. For ORR and CBR, point estimates and 95\% CIs were calculated using the Clopper-Pearson method. 
rwPFS, TTF, OS, ORR, and CBR for subgroup analysis were calculated using the same statistical methods described for the whole population.

Subgroup analyses included study outcome assessments stratified according to Eastern Cooperative Oncology Group Performance Status (ECOG PS; unknown vs $0, \geq 1$ vs 0 ), central nervous system (CNS) metastases (yes vs no), visceral metastases (yes vs no), hormone receptor status (positive vs negative), HER2 status (IHC3+ vs IHC2+l ISH+ IHC unknown/ISH+), number of treatment lines before T-DM1 ( $\geq 2$ vs $<2$ ), history of pertuzumab treatment (yes vs no), best response to T-DM1 treatment (CR or PR vs others), and regimens after T-DM1 (anti-HER2 therapy vs without anti-HER2 therapy).

Missing values were not imputed. Statistical analyses were performed using SAS software, version 9.4 (SAS Institute Inc., Cary, NC, USA). All tests were two-sided, with $p<0.05$ considered statistically significant.

\section{Results}

\section{Study population}

Patient disposition is shown in Fig. 1. Briefly, 205 patients who had received T-DM1 within the study period of 1 January 2014 and 31 December 2018 were identified from the medical records search. After evaluation against the inclusion/exclusion criteria, 138 patients were registered. The main reason for exclusion was that the start date of post-T-DM1 treatment did not occur within the study period. Ten registered patients were excluded from the analysis and the main reason for exclusion was that patients did not meet the inclusion criteria after completing T-DM1 treatment. The data collected for the remaining 128 patients were analyzed; the analysis population with measurable lesions included 111 patients.

Table 1 shows the patients' characteristics as well as those stratified by regimen type following T-DM1 treatment and type of metastatic cancer, and those stratified by recurrent / de novo cancer are shown in Additional File 1. In the total analysis population, the median (range) age was 59.0 (27-84) years. All patients were female with HER2-positive breast cancer (IHC3+, $81 \%$ $[n=104])$, and $65 \%(n=83)$ were hormone receptorpositive. Recurrent disease was reported for $64 \%(n=82)$ of patients (total analysis population), and $36 \%(n=46)$ had de novo disease (defined as Stage IV [Any T + Any $\mathrm{N}+\mathrm{M} 1]$ or recurrence within 6 months of the start of initial treatment). Regarding prior anti-HER2 therapies before T-DM1 (total analysis population), trastuzumab, pertuzumab, and lapatinib had been received by $94 \%$ $(n=120), 56 \%(n=72)$, and $28 \%(n=36)$ of patients, respectively. Regarding prior chemotherapy, $84 \%(n=108)$ had received taxane-based therapy and $50 \%(n=64)$ had received anthracycline-based therapy (Table 1 ). The median (range) T-DM1 treatment duration was $5.1(0.0-$ 41.4) months. Forty-six (36\%) patients had a best tumor response of CR or PR with T-DM1 treatment. The most common reason for T-DM1 discontinuation was disease progression (80\%).

At the start of post-T-DM1 treatment, 67 patients (52\%) had an ECOG PS score of 0, 25 patients (20\%) had a score of 1 , nine patients $(7 \%)$ had a score of $\geq 2$, and 27 patients $(21 \%)$ had an unknown score. The metastatic sites at post-T-DM1 treatment initiation (total analysis population) were as follows: visceral $(70 \%, n=$ 89), skin/subcutaneous soft tissue/lymph node $(59 \%, n=$

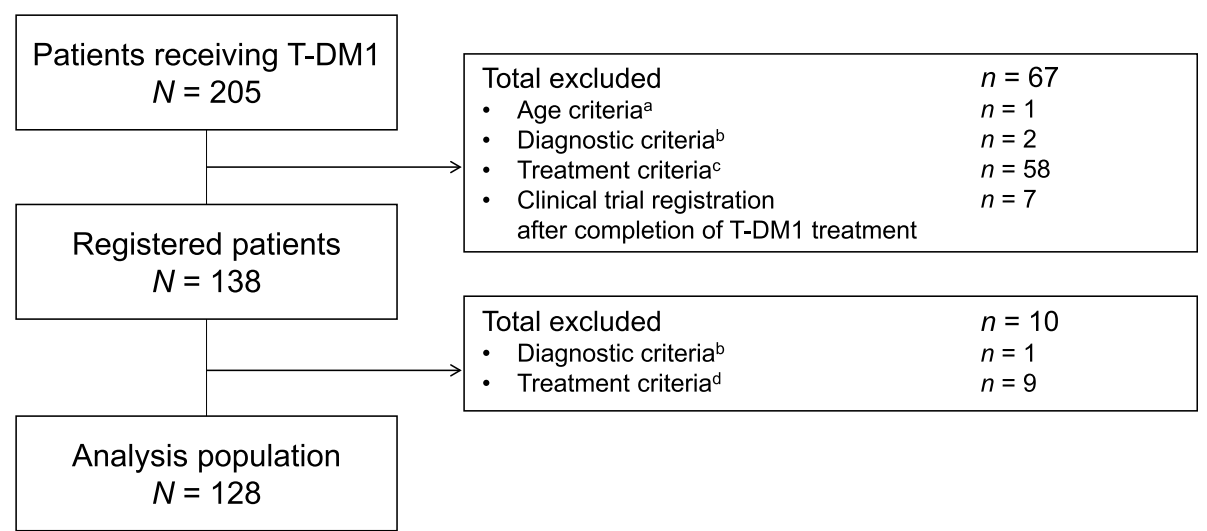

Fig. 1 Patient disposition. A Aged $\geq 20$ years at the start of drug therapy following T-DM1 treatment discontinuation. B Not pathologically diagnosed with unresectable and/or metastatic human epidermal growth factor receptor 2 (HER2)-positive breast cancer. C Did not start at least one line of drug therapy (anti-HER2 therapy, molecular targeted therapy, chemotherapy, and endocrine therapy) for unresectable and/or metastatic HER2-positive breast cancer following T-DM1 treatment discontinuation between 1 January 2014 and 31 December 2018. D At least one line of drug therapy (anti-HER2 therapy, molecular targeted therapy, chemotherapy, and endocrine therapy) was started for unresectable and/or metastatic HER2-positive breast cancer following T-DM1 treatment discontinuation, but the start date was 1 January 2019 or later. T-DM1 trastuzumab emtansine 
Table 1 Patient demographic characteristics according to regimen after T-DM1

\begin{tabular}{|c|c|c|c|}
\hline & All & Regimen after T-DN & \\
\hline & & $\begin{array}{l}\text { Anti-HER2 therapy } \\
(n=105)\end{array}$ & $\begin{array}{l}\text { Without anti-HER2 therapy } \\
(n=23)\end{array}$ \\
\hline Age (years) & & & \\
\hline Median (range) & $59.0(27-84)$ & $60.0(36-84)$ & $57.0(27-78)$ \\
\hline$\geq 60$ years & $62(48.4)$ & $54(51.4)$ & $8(34.8)$ \\
\hline ECOG PS & & & \\
\hline 0 & $67(52.3)$ & $57(54.3)$ & $10(43.5)$ \\
\hline 1 & $25(19.5)$ & $20(19.0)$ & $5(21.7)$ \\
\hline$\geq 2$ & $9(7.0)$ & $6(5.7)$ & $3(13.0)$ \\
\hline Unknown & $27(21.1)$ & $22(21.0)$ & $5(21.7)$ \\
\hline Hormone receptor status & & & \\
\hline Positive & $83(64.8)$ & $66(62.9)$ & $17(73.9)$ \\
\hline Negative & $43(33.6)$ & $38(36.2)$ & $5(21.7)$ \\
\hline Unknown & $2(1.6)$ & $1(1.0)$ & $1(4.3)$ \\
\hline HER2 status & & & \\
\hline $\mathrm{HCC}+$ & $104(81.3)$ & $83(79.0)$ & $21(91.3)$ \\
\hline $\mathrm{IHC2}+$ and $\mathrm{ISH}+$ & $21(16.4)$ & $19(18.1)$ & $2(8.7)$ \\
\hline $\mathrm{IHC}$ not performed and $\mathrm{ISH}+{ }^{a}$ & $3(2.3)$ & $3(2.9)$ & $0(0.0)$ \\
\hline Type of metastatic breast cancer & & & \\
\hline De novo ${ }^{b}$ & $46(35.9)$ & $39(37.1)$ & $7(30.4)$ \\
\hline Recurrent & $82(64.1)$ & $66(62.9)$ & $16(69.6)$ \\
\hline Disease-free interval (months), median (range) ${ }^{c}$ & $39.59(7.9-198.3)$ & 39.59 (7.9-198.3) & $42.00(9.9-193.8)$ \\
\hline Metastatic stie at initial metastatic diagnosis & & & \\
\hline Liver & $42(32.8)$ & $34(32.4)$ & $8(34.8)$ \\
\hline Lung & $36(28.1)$ & $33(31.4)$ & $3(13.0)$ \\
\hline Bone & $44(34.4)$ & $37(35.2)$ & $7(30.4)$ \\
\hline Peritoneal dissemination & $7(5.5)$ & $6(5.7)$ & $1(4.3)$ \\
\hline Ascites & $0(0.0)$ & $0(0.0)$ & $0(0.0)$ \\
\hline CNS & $5(3.9)$ & $5(4.8)$ & $0(0.0)$ \\
\hline Skin/subcutaneous soft tissues & $17(13.3)$ & $14(13.3)$ & $3(13.0)$ \\
\hline Lymph nodes & $60(46.9)$ & $50(47.6)$ & $10(43.5)$ \\
\hline Others & $6(4.7)$ & $5(4.8)$ & $1(4.3)$ \\
\hline Drug therapy prior to T-DM1 treatment & & & \\
\hline Anti-HER2 therapy & & & \\
\hline Trastuzumab & $120(93.8)$ & 99 (94.3) & $21(91.3)$ \\
\hline Pertuzumab & $72(56.3)$ & $58(55.2)$ & $14(60.9)$ \\
\hline Lapatinib & $36(28.1)$ & $28(26.7)$ & $8(34.8)$ \\
\hline None & $6(4.7)$ & $5(4.8)$ & $1(4.3)$ \\
\hline Chemotherapy & & & \\
\hline Anthracycline-based & $64(50.0)$ & $50(47.6)$ & $14(60.9)$ \\
\hline Taxane-based & $108(84.4)$ & $88(83.8)$ & $20(87.0)$ \\
\hline Paclitaxel & $56(43.8)$ & $42(40.0)$ & $14(60.9)$ \\
\hline Docetaxel & $80(62.5)$ & $67(63.8)$ & $13(56.5)$ \\
\hline Neither anthracycline nor taxane & $19(14.8)$ & $16(15.2)$ & $3(13.0)$ \\
\hline
\end{tabular}


Table 1 Patient demographic characteristics according to regimen after T-DM1 (Continued)

\begin{tabular}{|c|c|c|c|}
\hline & \multirow{2}{*}{$\begin{array}{l}\text { All } \\
(N=128)\end{array}$} & \multicolumn{2}{|l|}{ Regimen after T-DM1 } \\
\hline & & $\begin{array}{l}\text { Anti-HER2 therapy } \\
(n=105)\end{array}$ & $\begin{array}{l}\text { Without anti-HER2 therapy } \\
(n=23)\end{array}$ \\
\hline Capecitabine/S-1 & $47(36.7)$ & $34(32.4)$ & $13(56.5)$ \\
\hline \multicolumn{4}{|c|}{ No. of chemotherapy treatments before T-DM1 in any setting } \\
\hline 0 & $13(10.2)$ & $11(10.5)$ & $2(8.7)$ \\
\hline 1 & $36(28.1)$ & $32(30.5)$ & $4(17.4)$ \\
\hline 2 & $25(19.5)$ & $21(20.0)$ & $4(17.4)$ \\
\hline$\geq 3$ & $54(42.2)$ & $41(39.0)$ & $13(56.5)$ \\
\hline \multicolumn{4}{|c|}{ Duration from initial metastatic diagnosis to the start of T-DM1 treatment (months) } \\
\hline Median (range) & $22.00(0.03-174.9)$ & - & - \\
\hline \multicolumn{4}{|l|}{ Best response with T-DM1 } \\
\hline$C R, P R$ & $46(35.9)$ & $42(40.0)$ & $4(17.4)$ \\
\hline SD, non-CR/non-PD, PD & $80(62.5)$ & $62(59.0)$ & $18(78.3)$ \\
\hline Unknown & $2(1.6)$ & $1(1.0)$ & $1(4.3)$ \\
\hline \multicolumn{4}{|l|}{ Duration of T-DM1 treatment (months) } \\
\hline Median (range) & $5.09(0.03-41.4)$ & $5.78(0.7-41.4)$ & $2.33(0.03-26.5)$ \\
\hline$<6$ months & $74(57.8)$ & $54(51.4)$ & $20(87.0)$ \\
\hline$\geq 6$ to $<12$ months & $30(23.4)$ & $29(27.6)$ & $1(4.3)$ \\
\hline$\geq 12$ months & $24(18.8)$ & $22(21.0)$ & $2(8.7)$ \\
\hline \multicolumn{4}{|l|}{ Reason for T-DM1 treatment discontinuation } \\
\hline Disease progression & $102(79.7)$ & $82(78.1)$ & $20(87.0)$ \\
\hline Toxicity & $21(16.4)$ & $19(18.1)$ & $2(8.7)$ \\
\hline Other & $5(3.9)$ & $4(3.8)$ & $1(4.3)$ \\
\hline \multicolumn{4}{|c|}{ Metastatic site at start of drug therapy after T-DM1 treatment discontinuation } \\
\hline Viscera & $89(69.5)$ & $72(68.6)$ & $17(73.9)$ \\
\hline Skin/subcutaneous soft tissues/lymph nodes & $76(59.4)$ & $64(61.0)$ & $12(52.2)$ \\
\hline Bone & $53(41.4)$ & $42(40.0)$ & $11(47.8)$ \\
\hline CNS & $17(13.3)$ & $15(14.3)$ & $2(8.7)$ \\
\hline Other & $9(7.0)$ & $8(7.6)$ & $1(4.3)$ \\
\hline
\end{tabular}

Data are $n$ (\%) unless otherwise indicated

aThe study protocol states that "IHC3+ or IHC2+/ISH+ tumors are defined as HER2-positive". However, at the case review meeting, it was determined that study patients with "IHC not performed and ISH+" who underwent anti-HER2 therapy were to be regarded as HER2-positive

${ }^{b}$ Defined as Stage IV (Any T + Any N + M1) or recurrence within 6 months after the start of initial treatment

${ }^{\mathrm{C}} \mathrm{A}$ single missing case was excluded from recurrent cases

CNS central nervous system, CR complete response, ECOG PS Eastern Cooperative Oncology Group Performance Status, HER2 human epidermal growth factor receptor 2, IHC immunohistochemistry, ISH in situ hybridization, $P D$ progressive disease, $P R$ partial response, $S D$ stable disease, $T$-DMI trastuzumab emtansine

76), bone (41\%, $n=53)$, and CNS (13\%, $n=17)$. Overall, characteristics in the population with measurable lesions were similar to those in the total analysis population.

\section{Treatment regimens}

Treatment regimens after T-DM1 (total analysis population) are shown in Table 2 . Among the 128 patients analyzed, 105 (82\%) patients received anti-HER2 therapy and $23(18 \%)$ patients received regimens without anti-HER2 therapy. The following treatment regimens were used for patients who received anti-HER2 therapy: pertuzumabcontaining therapy (28\%) including a combination of pertuzumab with trastuzumab \pm chemotherapy or endocrine therapy, trastuzumab-containing therapy (excluding pertuzumab) (27\%), and lapatinib + capecitabine therapy (27\%).

\section{Efficacy outcomes}

The median (range) follow-up time was 15.5 (0.5-57.4) months. The median rwPFS ( $95 \% \mathrm{CI}$; number of events) was 5.7 months $(4.8-6.9 ; 109)$ (Fig. 2A) and the median TTF (95\% CI; number of events) was 5.6 months (4.6$6.4 ; 117)$. The median OS (95\% CI; number of events) was 22.8 months $(18.2-32.4$; 65) (Fig. 2B). Among the 
Table 2 Treatment regimen stratified by whether the patient was treated with or without anti-HER2 therapy

\begin{tabular}{ll}
\hline & $\begin{array}{l}\text { All } \\
(\boldsymbol{N}=\mathbf{1 2 8})\end{array}$ \\
\hline Anti-HER2 therapy & $105(82.0)$ \\
Trastuzumab + pertuzumab + chemotherapy & $32(25.0)$ \\
Trastuzumab + pertuzumab + endocrine therapy & $1(0.8)$ \\
Trastuzumab + pertuzumab & $3(2.3)$ \\
Trastuzumab + chemotherapy & $16(12.5)$ \\
Trastuzumab + endocrine therapy & $9(7.0)$ \\
Trastuzumab alone & $10(7.8)$ \\
Lapatinib + capecitabine & $34(26.6)$ \\
Regimens without anti-HER2 therapy & $23(18.0)$ \\
Bevacizumab + paclitaxel & $12(9.4)$ \\
Other chemotherapy & $5(3.9)$ \\
Everolimus + exemestane & $2(1.6)$ \\
Endocrine alone & $4(3.1)$ \\
\hline Data are $n$ (\%) \\
HER2 human epidermal growth factor receptor 2
\end{tabular}

111 patients with measurable lesions, one patient (0.9\%) achieved CR and 24 (22\%) achieved PR, with an ORR of 23\% (25/111; 95\% CI: 15.1-31.4). SD was achieved in 34 patients (31\%), 44 had PD (40\%), and eight (7\%) had an unknown response. The CBR (95\% CI) was 48\% (38.856.7).

\section{Subgroup analysis}

Subgroup analysis revealed that rwPFS was longer (per the $95 \% \mathrm{CI}$ ) in patients with the following characteristics: an ECOG PS score of 0 (6.7 months [95\% CI: 5.47.4]) vs an ECOG PS score of $\geq 1$ (3.9 months [95\% CI: 2.1-5.8]), HER2 IHC 3+ (6.2 months [95\% CI: 5.1-7.1]) vs IHC $2+/ \mathrm{ISH}+$ and IHC unknown/ISH+ (3.9 months
[95\% CI: 2.1-6.7]), no history of pertuzumab treatment (7.1 months [95\% CI: 5.7-7.9]) vs history of pertuzumab treatment (4.9 months [95\% CI: 4.0-5.8]), and antiHER2 therapy (6.3 months [95\% CI: 5.1-7.2]) vs regimens without anti-HER2 therapy (4.8 months [95\% CI: 1.9-5.9]). Other subgroup outcomes of TTF, OS, ORR, and CBR are shown in Additional File 1.

\section{Exploratory analysis of clinical factors associated with rwPFS}

To determine clinical variables associated with better median rwPFS, univariate and multivariate Coxregression analyses were performed. An ECOG PS score of 0 ( $\geq 1$ vs 0 : hazard ratio $1.81,95 \% \mathrm{CI} ; 1.16-2.84$ ), recurrent type $(\mathrm{HR}=0.68,95 \% \mathrm{CI} ; 0.42-0.97)$, a HER2 immunohistochemistry score of $3+(\mathrm{HR}=0.52,95 \% \mathrm{CI}$; 0.31-0.86), more than 12 months duration of T-DM1 treatment ( $\geq 12$ months vs $<6$ months: $\mathrm{HR}=0.55,95 \%$ CI; 0.32-0.96), and anti-HER2 therapy ( $\mathrm{HR}=0.48,95 \%$ CI; 0.28-0.83) were identified as independent variables for rwPFS in both analyses (Table 3). Kaplan-Meier curves for each subgroup are shown in Fig. 3A-E. The median rwPFS for anti-HER2 therapy was significantly better than that of regimens without anti-HER2 therapy $(p=0.004$, Fig. 3A). Interestingly and unexpectedly, rwPFS tended to be shorter in the de novo type than recurrent type $(p=0.058)$ (Fig. 3E).

\section{Discussion}

In this multicenter, retrospective, observational study, we found that among 128 patients with HER2-positive metastatic breast cancer in real-world clinical practice, $82 \%$ were treated with post-T-DM1 regimens that included anti-HER2 therapy and $18 \%$ were treated with post-T-DM1 regimens without anti-HER2 therapy. We report a median rwPFS and OS for post-T-DM1 therapy

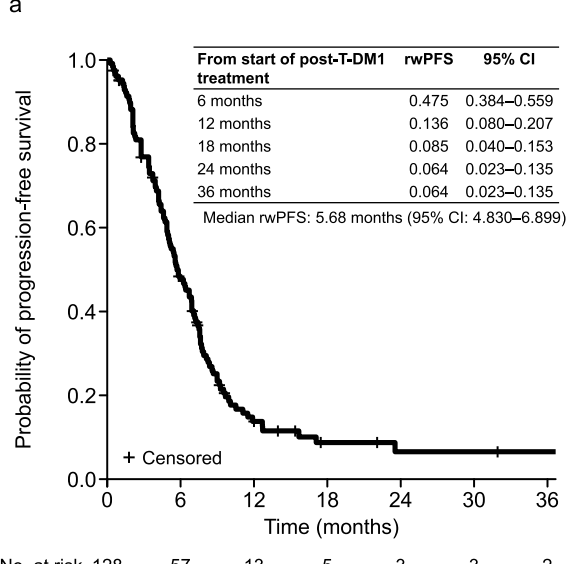

b

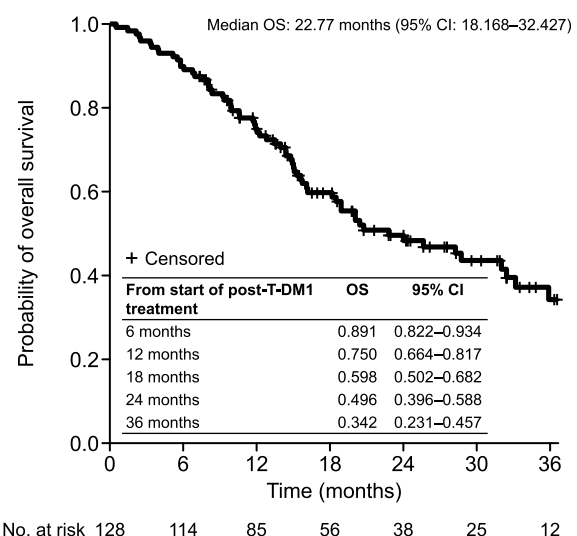

Fig. 2 Product-limit survival estimates for $(\mathbf{A})$ real-world progression-free survival and (B) overall survival. Cl confidence interval, OS overall survival, rWPFS real-world progression-free survival 
Table 3 Univariate/multivariate Cox-regression analysis for real-world progression-free survival

\begin{tabular}{|c|c|c|c|c|c|c|}
\hline & \multicolumn{2}{|c|}{$\begin{array}{l}\text { Univariate } \\
(N=128)\end{array}$} & \multicolumn{4}{|c|}{$\begin{array}{l}\text { Multivariate }^{a} \\
(N=126)\end{array}$} \\
\hline & $\mathrm{HR}^{\mathrm{b}}$ & $95 \% \mathrm{Cl}$ & $p$ value & $\mathrm{HR}^{\mathrm{b}}$ & $95 \% \mathrm{Cl}$ & $p$ value \\
\hline \multicolumn{7}{|l|}{ Age (years) } \\
\hline$\geq 60$ vs $<60$ & 0.70 & $0.478-1.024$ & 0.066 & - & - & - \\
\hline \multicolumn{7}{|l|}{ ECOG PS } \\
\hline Unknown vs 0 & 1.22 & $0.748-1.991$ & 0.425 & 0.95 & $0.555-1.621$ & 0.848 \\
\hline$\geq 1$ vs 0 & 1.81 & $1.161-2.824$ & 0.009 & 1.81 & $1.158-2.843$ & 0.009 \\
\hline Recurrent / de novo, recurrent vs de novo & 0.68 & $0.461-1.017$ & 0.061 & 0.64 & $0.423-0.968$ & 0.034 \\
\hline CNS metastasis, yes vs no & 1.02 & $0.577-1.790$ & 0.956 & - & - & - \\
\hline Visceral metastasis, yes vs no & 1.08 & $0.715-1.623$ & 0.724 & - & - & - \\
\hline Hormone receptor status, positive vs negative & 0.97 & $0.650-1.449$ & 0.883 & - & - & - \\
\hline HER2 status, IHC 3+ vs IHC 2+/ISH + or IHC unknown/ISH+ & 0.60 & $0.374-0.961$ & 0.034 & 0.52 & $0.307-0.864$ & 0.012 \\
\hline Number of treatment lines before T-DM1 treatment, $\geq 2 \mathrm{vs}<2$ & 1.01 & $0.688-1.491$ & 0.949 & - & - & - \\
\hline History of pertuzumab treatment, yes vs no & 1.55 & $1.054-2.286$ & 0.026 & 1.28 & $0.855-1.903$ & 0.232 \\
\hline History of lapatinib treatment, yes vs no & 0.94 & $0.620-1.439$ & 0.789 & - & - & - \\
\hline \multicolumn{7}{|l|}{ Regimen with anthracyclines and/or taxanes before T-DM1 } \\
\hline Yes (both) vs no (both) & 1.38 & $0.784-2.421$ & 0.265 & - & - & - \\
\hline Yes (either one) vs no (both) & 1.57 & $0.876-2.810$ & 0.130 & - & - & - \\
\hline \multicolumn{7}{|l|}{ Best response during T-DM1 treatment } \\
\hline$C R$ or $P R$ vs $S D$, non-CR/non-PD, $P D$, or unknown & 0.70 & $0.468-1.040$ & 0.077 & - & - & - \\
\hline \multicolumn{7}{|l|}{ Duration of T-DM1 treatment } \\
\hline$\geq 12$ months vs $<6$ months & 0.57 & $0.339-0.959$ & 0.034 & 0.55 & $0.318-0.959$ & 0.035 \\
\hline $6-12$ months vs $<6$ months & 0.60 & $0.377-0.940$ & 0.026 & 0.65 & $0.401-1.064$ & 0.087 \\
\hline \multicolumn{7}{|l|}{ Duration from the last day of T-DM1 to the start of the next regimen } \\
\hline$\geq 2$ months vs $<1$ month & 0.71 & $0.405-1.245$ & 0.233 & - & - & - \\
\hline $1-2$ months vs $<1$ month & 1.28 & $0.843-1.942$ & 0.247 & - & - & - \\
\hline \multicolumn{7}{|l|}{ Regimens after T-DM1 } \\
\hline Anti-HER2 therapy vs without anti-HER2 therapy & 0.50 & $0.305-0.814$ & 0.005 & 0.48 & $0.282-0.826$ & 0.008 \\
\hline
\end{tabular}

of 5.7 and 22.8 months, respectively; patients with measurable lesions had an ORR of $23 \%$. Both the PFS and ORR were similar to the data previously reported in the control arms of several recent global clinical trials, including the NALA, SOPHIA, monarcHER, and HER2CLIMB studies [23-27].

In the present study, a subgroup analysis revealed that treatment regimens including anti-HER2 therapy achieved better outcomes than those that did not include anti-HER2 therapy. Several previous studies have reported a benefit for trastuzumab beyond progression strategy [28, 29]. A report from Germany of patients with HER2-positive early and advanced breast cancer found that continuing trastuzumab treatment, combined with capecitabine beyond progression, significantly improved ORR and PFS compared with capecitabine treatment alone, which supports the continued use of anti-HER2 therapy [28]. Additionally, the findings from a retrospective review of patients who received trastuzumab for HER2-positive metastatic breast cancer showed that PFS on first-line trastuzumab-based therapy was a clinically relevant predictive factor for OS when patients were treated with trastuzumab after progression [29]. A retrospective study conducted in the US [30] investigated the clinical outcomes of lapatinib treatment in patients who had been treated with pertuzumab + trastuzumab and/or T-DM1; those patients had a TTF of 6.0 months, which is comparable with the rwPFS reported in our study. In the most recent Clinical Practice Guidelines for systemic treatment of breast cancer 


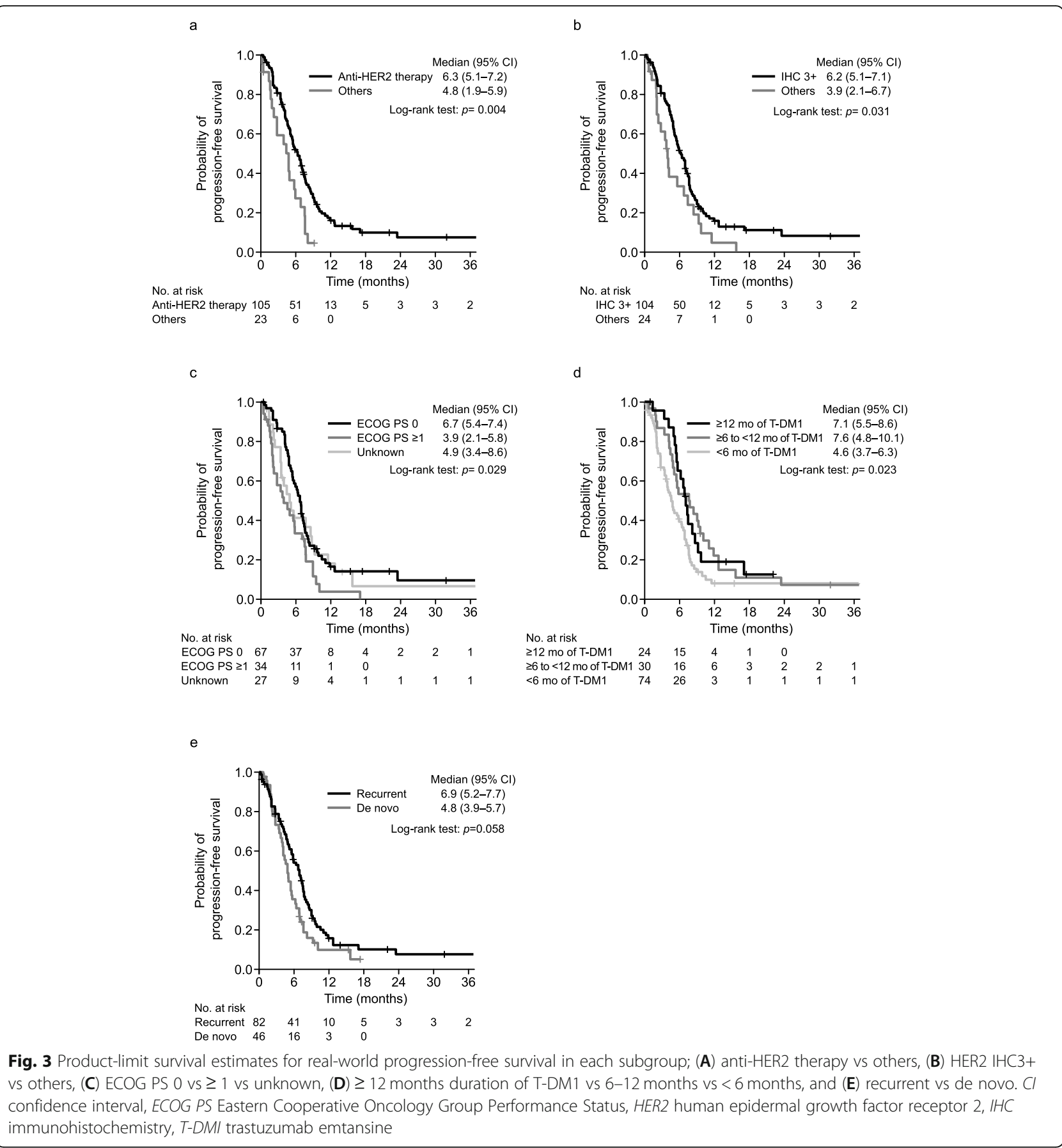

(Japanese Breast Cancer Society; 2018 edition [31]), continuation of anti-HER2 therapy is recommended as third-line or later in metastatic settings. The results of our study suggest that continuation of anti-HER2 therapy is an important option after T-DM1 treatment; therefore, this study supports the recent clinical guidelines from the Japanese Breast Cancer Society [31].

This study revealed that even among patients with HER2-positive breast cancer in Japan, some patients receive drug regimens without anti-HER2 therapy. For patients who had discontinued T-DM1 treatment after less than 6 months, there was a tendency to select a regimen without any anti-HER2 therapy, which is associated with shorter rwPFS post-T-DM1. Unlike in the West $[32,33]$, bevacizumab is approved in Japan for inoperable or recurrent breast cancer [34]. Bevacizumab + paclitaxel was the most frequently prescribed treatment (12/ 23 patients) as chemotherapy in combination with bevacizumab, which can be used not only as first-line in Japan but also as second-line or greater chemotherapy 
[31]. Among the available treatment options in Japan, this suggests that physicians are selecting bevacizumab + paclitaxel for patients with tumors that have low sensitivity to anti-HER2 therapy, based on the evidence from studies conducted in patients with HER2-negative tumors [35].

Our study showed better rwPFS in patients who had recurrent vs de novo cancer. Possible reasons for the high malignancy of de novo tumors are as follows: (1) the proportion of patients with liver metastasis, which is considered high risk at the time of initial metastasis diagnosis, was high; and (2) a high proportion of patients had brain metastasis at the time that post-T-DM1 treatment was initiated. Our study did not collect data related to tumor size or other factors, so further exploration of this is needed.

An ECOG PS of 0 (vs $\geq 1$ ) and tumors that were IHC3+ for HER2 (vs IHC2+/ISH+ and IHC unknown/ $\mathrm{ISH}+$ ) have been reported as prognostic factors [36, 37]. It is known that ECOG PS is related to the continuation of treatment and that high HER2 expression is related to sensitivity to anti-HER2 therapy. In the comparator group of our study, efficacy was still insufficient (median rwPFS: 3.9 months) and new treatment options are needed. The HER2CLIMB study recently reported that the addition of tucatinib to trastuzumab and capecitabine improved both PFS and OS in heavily pretreated patients with HER2-positive breast cancer, including those with CNS metastases [26]. Data from the DEST INY-Breast01 study showed that trastuzumab deruxtecan provided sustained antitumor activity in a population of patients with heavily pretreated HER2-positive metastatic breast cancer [38]. The PRECIOUS trial (NCT02514681) is currently being conducted by the Japan Breast Cancer Research Group to evaluate the efficacy of pertuzumab re-treatment in patients with HER2positive metastatic breast cancer previously treated with pertuzumab + trastuzumab + chemotherapy [39]. Therefore, to improve patient outcomes and prolong survival, clinicians must continue to evaluate new treatments and expand their knowledge of how treatment sequencing may impact subsequent efficacy.

This study had several limitations. As with any retrospective observational study, we relied on accurate record-keeping from treating physicians. Additionally, the sample size was limited and the study only included data from Japanese patients at five study sites; therefore, the results may not be entirely representative of the general population, potentially limiting their generalizability. However, as mentioned above, these data were collected at core cancer treatment hospitals. The data presented herein are specific to the real-world setting in Japan and these findings should be interpreted carefully in the case of real-world settings in other countries. Larger studies would be useful to confirm these findings and to expand on the subgroup analyses.

\section{Conclusion}

We conclude that the results of this study represent the real-world treatment patterns and outcomes of post-TDM1 therapy in Japan for patients with unresectable and/or metastatic HER2-positive breast cancer. Our results showed that continuation of anti-HER2 treatment and higher expression of HER2 were important factors for longer rwPFS, even in later lines of therapy for patients with HER2-positive breast cancer. However novel therapeutic options are still needed to further improve both PFS and OS in the real world. Future studies clarifying the real-world treatment situation for new antiHER2 therapies for HER2-positive breast cancer are expected.

\section{Abbreviations \\ CBR: Clinical benefit rate; Cl: Confidence interval; CR: Complete response; ECOG PS: Eastern Cooperative Oncology Group Performance Status; ER: Estrogen receptor; HER2: Human epidermal growth factor receptor 2; HR: Hazard ratio; IHC: Immunohistochemistry; ISH: In situ hybridization; KBCSG-TR: Kinki Breast Cancer Study Group-Translational Research; ORR: Objective response rate; OS: Overall survival; PD: Progressive disease; PFS: Progression-free survival; PR: Partial response; RECIST: Response Evaluation Criteria in Solid Tumors; RR: Response rate; rwPFS: Real-world progression-free survival; SD: Stable disease; T-DM1: Trastuzumab emtansine; TTF: Time-to-treatment failure}

\section{Supplementary Information}

The online version contains supplementary material available at https://doi. org/10.1186/s12885-021-08504-1

Additional file 1: Supplementary Text 1. Study outcomes.

Supplementary Table 1. Patient demographic characteristics (total analysis population and according to recurrent or de novo type). Supplementary Table $\mathbf{2}$. Analysis of outcomes according to subgroup.

\section{Acknowledgements}

We thank Sarah Bubeck, PhD, of Edanz Pharma for providing medical writing support, which was funded by Daiichi Sankyo Co., Ltd. Study support, editorial support, and publication management was provided by Daisuke Kuroki of Daiichi Sankyo Co., Ltd., Tokyo, Japan.

\section{Authors' contributions}

TN, TYO, HY, NK, MT, SO, SJK, HK, NY, TYa, TT, and NM contributed to the study conception and design. Material preparation, data collection, and analysis were performed by HK, NY, and TYa. The first draft of the manuscript was written by TN, NM, TYo, and TT. All authors commented on previous versions of the manuscript. All authors read and approved the final manuscript.

Funding

This study was funded by Daiichi Sankyo Co., Ltd.

Availability of data and materials

The datasets used and/or analyzed during the current study are available from the corresponding author upon reasonable request. 


\section{Declarations}

\section{Ethics approval and consent to participate}

This study was conducted in accordance with the ethical standards found in the 1964 Declaration of Helsinki, the Ethical Guidelines for Medical and Health Research Involving Human Subjects, and in compliance with the study protocol and all applicable local and national ethical guidelines. This study was approved in ethic screening committee of Osaka Prefectural Hospital Organization Osaka International Cancer Institute. As a non-interventional study with no invasive procedures or human-derived specimens, informed consent was neither required nor obtained from study participants. Participants were able to opt out of the study.

\section{Consent for publication}

Not applicable.

\section{Competing interests}

TN has received speakers' bureau fees from Chugai Pharma, AstraZeneca, El Lilly, Novartis, Takeda, Taiho Pharmaceutical, and Daiichi Sankyo. TYo has received consulting or advisory fees from Daiichi Sankyo and speakers' bureau fees from Chugai Pharma and Novartis. NM has received honoraria from Chugai Pharma, AstraZeneca, Pfizer, Eisai, Eli Lilly, and Takeda; research funding from Chugai Pharma, AstraZeneca, Kyowa Hakko Kirin, MSD, Novartis, Pfizer, Eli Lilly, Eisai, and Daiichi Sankyo; and has a leadership role at the Japan Breast Cancer Research Group. SO has received honoraria from Chugai Pharma, Pfizer, and Eisai. SJK has received honoraria and research funding from Daiichi Sankyo. MT has received honoraria from AstraZeneca, Eisai, Pfizer, Eli Lilly, Chugai Pharma, and Nippon Kayaku; and research funding from Taiho Pharmaceutical, Kyowa Hakko Kirin, Eisai, and Nippon Kayaku. TT is an employee of Daiichi Sankyo. NY has an immediate family member who is an employee of and has owned stock of held ownership interest in Bayer Yakuhin. NK, HK, HY, and TYa have no conflicting interests to declare.

\section{Author details}

'Department of Breast and Endocrine Surgery, Osaka International Cancer Institute, 3-1-69, Otemae, Chuo-ku, Osaka 541-8567, Japan. ${ }^{2}$ Department of Breast and Endocrine Surgery, Graduate School of Medicine, Osaka University, 2-2-E-10 Yamadaoka, Suita City, Osaka 565-0871, Japan. ${ }^{3}$ Department of Surgery, Breast Oncology, National Hospital Organization Osaka National Hospital, 2-1-14, Hoenzaka, Chuo-ku, Osaka 540-0006, Japan. ${ }^{4}$ Department of Breast Surgery, National Hospital Organization Hokkaido Cancer Center, 2-3-54, Kikusui 4-jo Shiroishi-ku, Sapporo, Hokkaido 003-0804, Japan. ${ }^{5}$ Department of Breast Surgery, Hiroshima City Hiroshima Citizens Hospital, 7-33 Motomachi, Naka-ku, Hiroshima 730-8518, Japan. ${ }^{6}$ Present address: Ohtani Shoichiro Breast Clinic, 4-18-101, Hatchobori, Naka-ku, Hiroshima 730-0013, Japan. 'Department of Medical Innovation, Osaka University Hospital, 2-15, Yamadaoka, Suita, Osaka 565-0871, Japan. ${ }^{8}$ Oncology Medical Science Department, Daiichi Sankyo Co., Ltd., 3-5-1, Nihonbashi-honcho, Chuo-ku, Tokyo 103-8426, Japan.

Received: 17 November 2020 Accepted: 10 June 2021

Published online: 09 July 2021

\section{References}

1. Onitilo AA, Engel JM, Greenlee RT, Mukesh BN. Breast cancer subtypes based on ER/PR and Her2 expression: comparison of clinicopathologic features and survival. Clin Med Res. 2009;7(1-2):4-13. https://doi.org/1 $0.3121 / \mathrm{cmr} .2008 .825$

2. Gonzalez-Angulo AM, Litton JK, Broglio KR, Meric-Bernstam F, Rakkhit R, Cardoso F, et al. High risk of recurrence for patients with breast cancer who have human epidermal growth factor receptor 2-positive, node-negative tumors $1 \mathrm{~cm}$ or smaller. J Clin Oncol. 2009;27(34):5700-6. https://doi.org/1 0.1200/JCO.2009.23.2025

3. Slamon DJ, Clark GM, Wong SG, Levin WJ, Ullrich A, McGuire WL. Human breast cancer: correlation of relapse and survival with amplification of the HER-2/neu oncogene. Science. 1987;235(4785):177-82. https://doi.org/10.112 6/science.3798106

4. Slamon DJ, Godolphin W, Jones LA, Holt JA, Wong SG, Keith DE, et al. Studies of the HER-2/neu proto-oncogene in human breast and ovarian cancer. Science. 1989;244(4905):707-12. https://doi.org/10.1126/science.24 70152.

5. Harbeck N. Advances in targeting HER2-positive breast cancer. Curr Opin Obstet Gynecol. 2018;30(1):55-9. https://doi.org/10.1097/GCO. 0000000000000431.

6. Menard S, Fortis S, Castiglioni F, Agresti R, Balsari A. HER2 as a prognostic factor in breast cancer. Oncology. 2001;61(Suppl 2):67-72. https://doi.org/1 $0.1159 / 000055404$

7. Slamon DJ, Leyland-Jones B, Shak S, Fuchs H, Paton V, Bajamonde A, et al. Use of chemotherapy plus a monoclonal antibody against HER2 for metastatic breast cancer that overexpresses HER2. N Engl J Med. 2001; 344(11):783-92. https://doi.org/10.1056/NEJM200103153441101.

8. Maximiano S, Magalhaes P, Guerreiro MP, Morgado M. Trastuzumab in the treatment of breast cancer. BioDrugs. 2016;30(2):75-86. https://doi.org/10.1 007/s40259-016-0162-9.

9. Cameron D, Casey M, Press M, Lindquist D, Pienkowski T, Romieu CG, et al. A phase III randomized comparison of lapatinib plus capecitabine versus capecitabine alone in women with advanced breast cancer that has progressed on trastuzumab: updated efficacy and biomarker analyses. Breast Cancer Res Treat. 2008;112(3):533-43.

10. Geyer CE, Forster J, Lindquist D, Chan S, Romieu CG, Pienkowski T, et al. Lapatinib plus capecitabine for HER2-positive advanced breast cancer. N Engl J Med. 2006;355(26):2733-43. https://doi.org/10.1056/NEJMoa064320.

11. Baselga J, Cortes J, Kim SB, Im SA, Hegg R, Im YH, et al. Pertuzumab plus trastuzumab plus docetaxel for metastatic breast cancer. N Engl J Med. 2012;366(2):109-19. https://doi.org/10.1056/NEJMoa1113216.

12. Swain SM, Baselga J, Kim SB, Ro J, Semiglazov V, Campone M, et al. Pertuzumab, trastuzumab, and docetaxel in HER2-positive metastatic breast cancer. N Engl J Med. 2015;372(8):724-34. https://doi.org/10.1056/ NEJMoa1413513.

13. Verma S, Miles D, Gianni L, Krop IE, Welslau M, Baselga J, et al. Trastuzumab emtansine for HER2-positive advanced breast cancer. N Engl J Med. 2012; 367(19):1783-91. https://doi.org/10.1056/NEJMoa1209124.

14. Krop IE, Kim SB, Martin AG, LoRusso PM, Ferrero JM, Badovinac-Crnjevic T, et al. Trastuzumab emtansine versus treatment of physician's choice in patients with previously treated HER2-positive metastatic breast cancer (TH3RESA): final overall survival results from a randomised open-label phase 3 trial. Lancet Oncol. 2017;18(6):743-54. https://doi.org/10.1016/S1470-204 5(17)30313-3.

15. Krop IE, Kim SB, Gonzalez-Martin A, LoRusso PM, Ferrero JM, Smitt M, et al. Trastuzumab emtansine versus treatment of physician's choice for pretreated HER2-positive advanced breast cancer (TH3RESA): a randomised, open-label, phase 3 trial. Lancet Oncol. 2014;15(7):689-99. https://doi.org/1 0.1016/S1470-2045(14)70178-0.

16. Dieras V, Miles D, Verma S, Pegram M, Welslau M, Baselga J, et al. Trastuzumab emtansine versus capecitabine plus lapatinib in patients with previously treated HER2-positive advanced breast cancer (EMILIA): a descriptive analysis of final overall survival results from a randomised, openlabel, phase 3 trial. Lancet Oncol. 2017;18(6):732-42. https://doi.org/10.1016/ S1470-2045(17)30312-1.

17. Kagiyama N, Matsue $\mathrm{Y}$. The time-to-treatment concept in acute heart failure: lessons and implications from REALITY-AHF. Anatol J Cardiol. 2018;20(2): 125-9. https://doi.org/10.14744/AnatolJCardiol.2018.88935.

18. NCCN Clinical Practice Guidelines in Oncology: Breast Cancer. https://www. nccn.org/professionals/physician_gls/pdf/breast.pdf.

19. Hunter FW, Barker HR, Lipert B, Rothe F, Gebhart G, Piccart-Gebhart MJ, et al. Mechanisms of resistance to trastuzumab emtansine (T-DM1) in HER2positive breast cancer. Br J Cancer. 2020;122(5):603-12. https://doi.org/10.1 038/s41416-019-0635-y.

20. Yoshinami T, Hasegawa A, Fujisawa F, Kittaka N, Ishitobi M, Sugimoto N, et al. Abstract P4-22-24: a retrospective study about re-biopsy at disease progression on T-DM1. Cancer Res. 2017;77(4 Suppl):4 -22-24-P24-22-24.

21. Sakamoto G, Inaji H, Akiyama F, Haga S, Hiraoka M, Inai K, et al. General rules for clinical and pathological recording of breast cancer 2005. Breast Cancer. 2005;12(Suppl):S1-27.

22. Griffith SD, Tucker M, Bowser B, Calkins G, Chang CJ, Guardino E, et al. Generating real-world tumor burden endpoints from electronic health record data: comparison of RECIST, radiology-anchored, and cliniciananchored approaches for abstracting real-world progression in non-small cell lung cancer. Adv Ther. 2019;36(8):2122-36. https://doi.org/10.1007/s1232 5-019-00970-1. 
23. Saura C, Oliveira M, Feng Y-H, Dai M-S, Hurvitz SA, Kim S-B, et al. Neratinib + capecitabine versus lapatinib + capecitabine in patients with HER2+ metastatic breast cancer previously treated with $\geq 2$ HER2-directed regimens: Findings from the multinational, randomized, phase III NALA trial. J Clin Oncol. 2019;37(15_suppl):1002.

24. Rugo HS, Im S-A, Wright GLS, Escriva-de-Romani S, DeLaurentiis M, Cortes J, et al. SOPHIA primary analysis: A phase 3 (P3) study of margetuximab (M) + chemotherapy $(C)$ versus trastuzumab $(T)+C$ in patients (pts) with HER2+ metastatic (met) breast cancer (MBC) after prior anti-HER2 therapies (Tx). J Clin Oncol. 2019;37(15_suppl):1000.

25. Tolaney SM, Bourayou N, Goel S, Forrester T, André F. monarcHER: A phase 2 randomized open-label study of abemaciclib plus trastuzumab (T) with or without fulvestrant (F) compared to standard-of-care chemotherapy of physician's choice plus T in women with HR +, HER2+ advanced breast cancer. Ann Oncol. 2016:27:vi99.

26. Murthy RK, Loi S, Okines A, Paplomata E, Hamilton E, Hurvitz SA, et al. Tucatinib, trastuzumab, and capecitabine for HER2-positive metastatic breast cancer. N Engl J Med. 2020;382(7):597-609. https://doi.org/10.1056/NEJMoa1 914609.

27. A study of neratinib plus capecitabine versus lapatinib plus capecitabine in patients with HER2 + metastatic breast cancer who have received two or more prior HER2 directed regimens in the metastatic setting (NALA). https://clinicaltrials.gov/ct2/show/results/NCT01808573?view=results. Accessed 8 March 2020

28. von Minckwitz G, du Bois A, Schmidt M, Maass N, Cufer T, de Jongh FE, et al. Trastuzumab beyond progression in human epidermal growth factor receptor 2-positive advanced breast cancer: a german breast group 26/ breast international group 03-05 study. J Clin Oncol. 2009;27(12):1999-2006. https://doi.org/10.1200/JCO.2008.19.6618.

29. Rayson D, Lutes S, Walsh G, Sellon M, Colwell B, Dorreen M, et al. Trastuzumab beyond progression for HER2 positive metastatic breast cancer: progression-free survival on first-line therapy predicts overall survival impact. Breast J. 2014;20(4):408-13. https://doi.org/10.1111/tbj.12284.

30. Baez-Vallecillo L, Raghavendra AS, Hess KR, Barcenas CH, Moulder SL, Tripathy D, et al. Lapatinib activity in metastatic human epidermal growth factor receptor 2-positive breast cancers that received prior therapy with trastuzumab, pertuzumab, and/or ado-trastuzumab emtansine (T-DM1). Breast Cancer Res Treat. 2019;176(1):227-34. https://doi.org/10.1007/s10549018-05081-z

31. Shimoi T, Nagai SE, Yoshinami T, Takahashi M, Arioka H, Ishihara M, et al. The Japanese breast Cancer society clinical practice guidelines for systemic treatment of breast cancer, 2018 edition. Breast Cancer. 2020;27(3):322-31. https://doi.org/10.1007/s12282-020-01085-0.

32. AVASTIN (bevacizumab) [prescribing information] https://www.accessdata. fda.gov/drugsatfda_docs/label/2014/125085s301 lbl.pdf. .

33. Union Register of medicinal products for human use. Product information: Avastin. https://ec.europa.eu/health/documents/community-register/html/ h300.htm. Accessed 11 March 2021.

34. Avastin (bevacizumab) [package insert]. https://www.info.pmda.go.jp/go/pa ck/4291413A1022_1_23/. Accessed 11 March 2021. [In Japanese].

35. Wang $X$, Huang C, Li M, Gu Y, Cui Y, Li Y. The efficacy of bevacizumab plus paclitaxel as first-line treatment for HER2-negative metastatic breast cancer: a meta-analysis of randomized controlled trials. Tumour Biol. 2014;35(5): 4841-8. https://doi.org/10.1007/s13277-014-1635-4.

36. Gamucci T, Pizzuti L, Natoli C, Mentuccia L, Sperduti I, Barba M, et al. A multicenter REtrospective observational study of first-line treatment with PERtuzumab, trastuzumab and taxanes for advanced HER2 positive breast cancer patients. RePer Study. Cancer Biol Ther. 2019;20(2):192-200.

37. Vici P, Pizzuti L, Michelotti A, Sperduti I, Natoli C, Mentuccia L, et al. A retrospective multicentric observational study of trastuzumab emtansine in HER2 positive metastatic breast cancer: a real-world experience. Oncotarget. 2017;8(34):56921-31.

38. Modi S, Saura C, Yamashita T, Park YH, Kim SB, Tamura K, et al. Trastuzumab deruxtecan in previously treated HER2-positive breast cancer. N Engl J Med. 2020;382(7):610-21. https://doi.org/10.1056/NEJMoa1914510.

39. Yamamoto $Y$, Iwata $H$, Ueno T, Taira N, Kashiwaba M, Takahashi M, et al. A randomized, open-label, phase III trial of pertuzumab retreatment in HER2positive locally advanced/metastatic breast cancer patients previously treated with pertuzumab, trastuzumab and chemotherapy: the Japan breast Cancer research group-M05 PRECIOUS study. Jpn J Clin Oncol. 2018;48(9): 855-9. https://doi.org/10.1093/jjco/hyy097.

\section{Publisher's Note}

Springer Nature remains neutral with regard to jurisdictional claims in published maps and institutional affiliations.

\section{Ready to submit your research? Choose BMC and benefit from:}

- fast, convenient online submission

- thorough peer review by experienced researchers in your field

- rapid publication on acceptance

- support for research data, including large and complex data types

- gold Open Access which fosters wider collaboration and increased citations

- maximum visibility for your research: over $100 \mathrm{M}$ website views per year

At $\mathrm{BMC}$, research is always in progress.

Learn more biomedcentral.com/submissions 